\title{
Previously rewarding environments enhance incidental memory formation
}

\author{
Anatoly Shneyer ${ }^{1,2}$ and Avi Mendelsohn ${ }^{1,2}$ \\ ${ }^{1}$ Sagol Department for Neurobiology, University of Haifa, Haifa 3498838, Israel; ${ }^{2}$ Institute of Information Processing and Decision \\ Making, University of Haifa, Haifa 3498838, Israel
}

\begin{abstract}
Declarative memory performance is superior for items that were encoded in temporal proximity to reward delivery or expectancy. How reward-predicting contexts affect subsequent declarative memory formation in those contexts are, however, unknown. Using an ecological experimental setup in the form of a naturalistic driving simulator task, we examined the effect that previously rewarded environments may have on incidental memory formation. After driving in two distinct environments, one of which associated with monetary reward, participants drove again in the environments, which were embedded with unique images on billboards. A recognition test $24 \mathrm{~h}$ later demonstrated that incidental memory was superior for items presented in the reward-associated environment. These findings suggest that environmental cues imbued with incentive salience promote memory processes even in the absence of reward.
\end{abstract}

A burgeoning notion in the field of neural mechanisms of memory formation emphasizes the interactive nature of declarative and nondeclarative learning and memory systems. Accordingly, mediating factors involved in nondeclarative learning, such as reinforcement learning, may affect declarative memory formation (Poldrack et al. 2001; Gershman and Daw 2017). Indeed, declarative memory formation has been shown to benefit from conditions where information is encoded in temporal proximity to reward delivery (Murayama and Kitagami 2014). Such reward-related memory enhancements are thought to be supported by functional links between the reward circuitry and declarative memory systems (Lisman and Grace 2005), possibly mediated by dopaminergic input to the hippocampus (Shohamy and Adcock 2010). Specifically, incidental memory enhancement has been described for items presented immediately before reward-predicting cues (Murayama and Kuhbandner 2011; Murayama and Kitagami 2014), or concurrent with them (Wittmann et al. 2005, 2013), during reward anticipation (Adcock et al. 2006), as well as during (Axmacher et al. 2010), and immediately after (unexpected) reward delivery (Bunzeck et al. 2010; Spaniol et al. 2014). These studies suggest that information appearing in short proximity to rewarding outcomes or predictors has a better chance of consolidating into long-term memory. It is not yet clear whether contextual cues associated with rewarding outcomes can maintain a memory-facilitating effect at a later stage. Moreover, it is not clear whether and how the abovementioned findings relate to real-life situations that involve natural behavior in immersive environments.

The neural mechanisms that mediate the interactions between reward-related systems and declarative memory formation are starting to be revealed. Dopamine, a key mediator of rewardbased learning, was shown to play a modulatory role not only in implicit forms of learning but also in explicit, hippocampaldependent memory (Shohamy and Adcock 2010; Pennartz et al. 2011). In fact, input from dopaminergic midbrain neurons can induce long-term potentiation (LTP) in the hippocampus (Frey and Morris 1998; Li et al. 2003), and dopamine agonists, both in animals and in humans, were shown to facilitate performance in explicit memory tasks (Bach et al. 1999; Knecht et al. 2004; Martig and Mizumori 2011). Moreover, midbrain activity levels in hu-

\section{Corresponding author: amendels1@univ.haifa.ac.il}

Article is online at http://www.learnmem.org/cgi/doi/10.1101/lm.047886.118. mans, as detected in fMRI studies, are predictive of memory performance for reward-related cues (Wittmann et al. 2005), and dopamine binding potential in the hippocampus is positively correlated with memory performance for items encoded during periods of reward expectancy (Takahashi et al. 2008; Wittmann et al. 2013). Ventral striatum activation was recently shown to correlate with the extent of expectancy violations (or prediction errors) regarding semantic memory veracity, emphasizing the interaction between reward and declarative memory systems (Pine et al. 2018). How associations between reward and environmental contexts in ecological setups may affect subsequent declarative memory for incidental information is, however, unclear.

Cues that have been imbued with incentive value activate motivational systems, which in turn engender the potentiation of behaviors that were associated with similar rewards, a process that has been termed Pavlovian-to-instrumental transfer (PIT) (Dickinson and Balleine 1994). It is not known whether cues that have been associated with reward may similarly potentiate memory formation of information embedded within those cues at a future time. To examine this question, we constructed an ecological driving simulator setup, comprised of three stages. First, participants drove in both rewarding and nonrewarding contexts. Subsequently, they drove again in both contexts, devoid of rewarding outcomes, and were presented with a set of pictures embedded within billboards in both environments. Finally, a day later, they underwent a recognition memory test for the images presented previously.

Thirty participants took part in the experiment (mean age of $26.6 \pm 3.7 \mathrm{yr} ; 10$ males). All participants were healthy, had normal or corrected-to-normal vision, and provided written informed consent to participate in the study, for which they were remunerated. The experimental protocol was approved by the Institutional Review Board of the Department of Psychology at the University of Haifa. The driving task was conducted at the Institute of Information Processing and Decision Making (IIPDM) driving

(C) 2018 Shneyer and Mendelsohn This article is distributed exclusively by Cold Spring Harbor Laboratory Press for the first 12 months after the full-issue publication date (see http://learnmem.cshlp.org/site/misc/terms.xhtml). After 12 months, it is available under a Creative Commons License (AttributionNonCommercial 4.0 International), as described at http://creativecommons. org/licenses/by-nc/4.0/. 
simulator facility, located at the University of Haifa. The facility includes both the driving simulator and a screen on which the simulation is projected and is isolated for noise and other interferences (see Fig. 1). The virtual environment was constructed using the STISIM Drive software and was presented on a wide white screen. The simulator allows full control of the participants over the car's gas and brake pedals and steering wheel, which are situated on a student's chair 2.5 meters away from the screen. The virtual environments depicted below were created using the STISIM Drive software, designed to reflect real-life navigation without resemblances to actual routes. The entire driving task lasted $\sim 40 \mathrm{~min}$.

On Day 1, participants first underwent a short training phase in order to acquaint them with the simulator environment. This stage was comprised of both an urban and a rural landscape. Next, participants underwent a context-reward association phase, during which they were able to receive monetary reward (up to 30 Israeli Shekels-ILS, the equivalent of $\sim 8$ USD) while driving the simulator. Each participant drove in two distinct environments, one urban and one rural. The conditioning was based on the acquisition of an association between one visual context (e.g., rural) and a reward, and a separate association between the other visual context (e.g., urban) with a smaller reward. Each environment (context) included presentations of stars, golden and blue, which were situated along the driving route. Driving into the golden stars indicated that the participant had won an additional 1-ILS on top of the participation remuneration. Blue stars had no value, and were therefore considered neutral. Both contexts were defined by the percentage of rewarding stars (gold) versus unrewarding stars (blue). Accordingly, the rewarding context was comprised of
A Day1

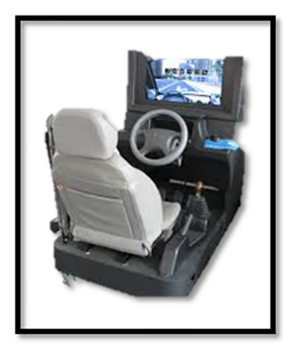

B Day2
Context-Reward association

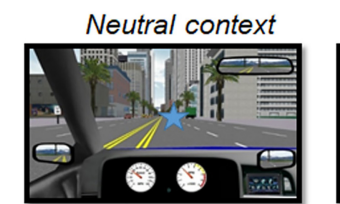

Immediately

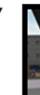

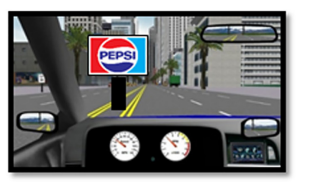

24 hours

1

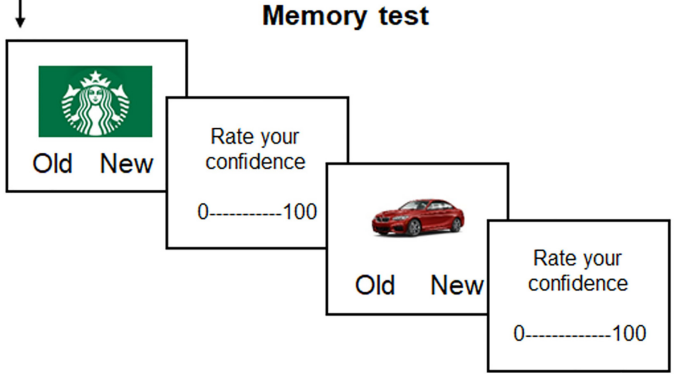

FICURE 1. Experimental design. ( $A$ ) During the context-reward association phase, participants drove in two distinct environments-an urban landscape and an open countryside, one of which was assigned as a "rewarded context" wherein they encountered mostly gold starts indicating monetary reward, and the other as a "neutral context," containing mostly blue stars (zero reward). Free driving was immediately followed, during which participants drove in both landscapes, this time devoid of reward. Embedded within both landscapes were unique images presented on billboards along the median divider or road shoulder of the route. $(B)$ One day later, participants returned to carry out a memory test, in which they were asked to indicate whether displayed images were presented the day before and indicate their confidence in their decision. (ILS) Israeli Shekel (Israeli currency).
$80 \%$ gold stars, while the neutral context was comprised of only $20 \%$ gold stars. For each participant, the order in which he/she enenvironment (context) and the rewarded context itself were assigned pseudo-randomly. Immediately after the association stage, participants drove again in both rural and urban landscapes, this time devoid of reward. During this task, they were presented with 40 neutral images (20 in each environment) which they were not asked to pay attention to (but were nonetheless prominent in the environment). The images were presented on billboards that appeared either in the median strip (the divider lane) or road shoulder, which gradually appeared larger on screen with the car's advancing. The assignment of images to context condition (previously rewarded/nonrewarded) was counter-balanced across participants, such that a given set of pictures was presented either in previously rewarding or nonrewarding contexts.

On Day 2, participants carried out a computerized memory test, whereby they were instructed to indicate whether or not presented images appeared in the driving phase. Eighty images were presented in total, 40 of which appeared during the free driving stage (20 in each environment) and 40 new ones. Participants were asked to answer for each image whether they recognized the image or not. Following each question, they were asked to ate their degree of confidence using a 1-9 visual analog scale (VAS). This memory task was designed and performed using E-Prime software. Immediately after the memory test, participants filled out a short questionnaire regarding subjective feelings and thoughts on the overall experience.

Memory performance was estimated by computing for each subject percentages of hits, misses, false alarms (FA) and correct rejections (CR) and plotting their means across the group. Direct statistical comparisons were carried out between hit percentages and miss percentages for images previously presented in the rewarded versus nonrewarded contexts, using Student's $t$-tests, as well as by carrying out Bayesian paired samples $t$-tests (Morey and Rouder 2011) implemented in JASP software (Version 0.9.0.1). For estimating the relationship between memory performance and confidence judgments in the two conditions of interest, hit rates versus FA rates were plotted as a function of confidence ratings, as common in adaptations of signal-dependent analysis schemes. Memory strength was further assessed by calculating d-prime $\left(\mathrm{d}^{\prime}\right)$ scores, by subtracting for each participant the normalized FA rate from hit rate $[z$ (hit rate) $-z$ (FA rate)]. Criterion values were calculated as well for estimating the participants' response biases in favor of "old/new" responses for rewarded versus nonrewarded conditions $[c=-0.5 \times$ $(z$ (hit rate) $+z$ (FA rate) $)]$. For both $\mathrm{d}^{\prime}$ and criterion indices, both individual data and group averages were plotted. The Bayesian paired sample analyses were performed by using either a nondirectional hypothesis (for hits versus misses regardless of contexts, and the early versus late hit percentages), or directional hypotheses for testing reward versus nonreward performance of d's, criterions, and hit percentages. 
A unique feature of the current task was the naturalistic experience that was afforded to participants by driving in an immersive setting (Plancher et al. 2013). Nearly all participants could identify that there were two distinctive environments (urban/rural) and perhaps surprisingly, none of them realized that there was a difference in the rewards given within each environment (i.e., the presented stars), indicating that any association made between reward and context was made without explicit knowledge. Furthermore, participants were asked about what they thought was the goal of the experiment. Virtually all of them answered that the prime objective was to test attention to details while driving. That is, participants did typically notice the images presented as signs, but nearly all did not realize they would be tested on them. Notably, the stars and images were not presented at the exact same location on the route-whereas the stars were placed on the road, the images appeared on the dividing lane or road shoulder.

On average, memory performance was considerably low (mean hit percentage $=40.08 \pm 3.12$ ), below-chance level (Fig. $2 \mathrm{~A})$. However, FA were even lower $(22.91 \pm 2.72)$, indicating that overall, participants responded positively more frequently to old versus new items $\left(t_{(29)}=5.08, P<0.0001\right.$; Bayes factor $\left(\mathrm{BF}_{10}\right)=$ 149.6). Testing memory performance for images presented in previously rewarded contexts versus nonrewarded contexts was performed by comparing hit percentages and FA percentages between rewarded and nonrewarded contexts. Hit percentages were found to increase for items presented in the previously rewarded contexts (means \pm SEs for hits rewarded and nonrewarded $=43.44 \pm 3.45$ and $36.83 \pm 3.53$, respectively, $t_{(29)}=2.09, P<0.05 ; \mathrm{BF}_{10}=2.51$ ), and a trend for increased misses for the nonrewarded context (misses rewarded $=55 \pm 3.72$ and misses nonrewarded $=60.5 \pm 3.59, t_{(29)}=$ $\left.-1.72, P=0.096 ; \mathrm{BF}_{10}=1.36\right)$. Notably, the enhanced memory for rewarded versus nonrewarded context was observed regardless of specific context, i.e., separately for the urban environment (mean hits percentage for rewarded versus nonrewarded: $43.33 \pm$ 4.77 versus $37.33 \pm 4.95$, respectively), and for the rural environment ( $43.33 \pm 5.16$ versus $36.33 \pm 5.22$ ), further strengthening the reward-related memory effect in two independent samples. These effects, however, were not statistically significant, possibly due to low sample sizes (N/2).

Plotting hit rates versus FA rates as a function of subjective confidence ratings (Fig. 2B) provided further evidence that at all confidence bins, mean hit rates were both higher than FA rates, and superior for rewarded versus nonrewarded contexts. Notably, even at high confidence assessments (leftmost area of the ROCs), hit rates were considerably low and the curves seem symmetrical, augmenting the notion that retrieval decisions here were based on familiarity rather than recollection (Yonelinas et al. 1996; Yonelinas 2001). D-prime ( $\left.\mathrm{d}^{\prime}\right)$ analysis revealed a significant difference between memory strength for images presented in the rewarded $($ mean $=0.71 \pm 0.13)$ versus nonrewarded $($ mean $=0.52 \pm 0.13)$ contexts $\left(t_{(29)}=2.14, P<0.05 ; \mathrm{BF}_{10}=3.303\right)$. These results demonstrate that memory for stimuli presented in the previously rewarded environments was superior in comparison to memory for stimuli presented in previously nonrewarded contexts (Fig. 2C). As shown in Figure 2C, most participants exhibited higher memory performance $\left(\mathrm{d}^{\prime}\right)$ scores for the pictures previously presented in the rewarded versus nonrewarded context (dots falling under the line). Corroborating the above findings, criterion values were significantly lower in the rewarded context $(0.54 \pm 0.09)$ versus the nonrewarded condition $\left(0.64 \pm 0.09 ; t_{(29)}=-2.24, P<0.05 ; \mathrm{BF}_{10}=\right.$ $3.302)$, indicating an increased tendency to answer "yes" in responses to images presented in the previously rewarded context.

To examine the possibility that the memory effect for the rewarding context condition was time-dependent, i.e., affecting the

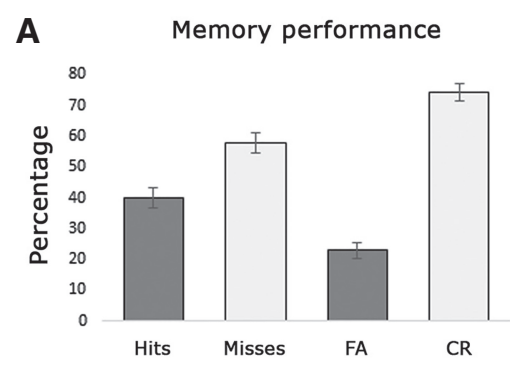

A

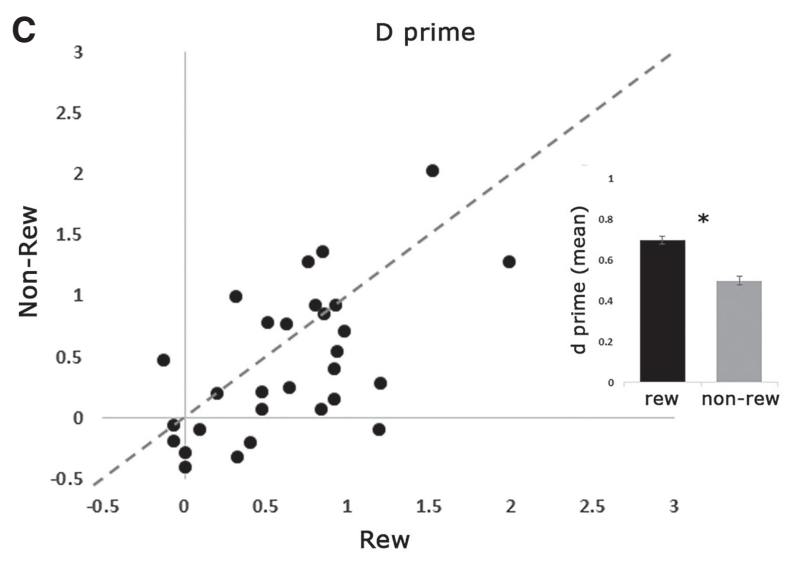

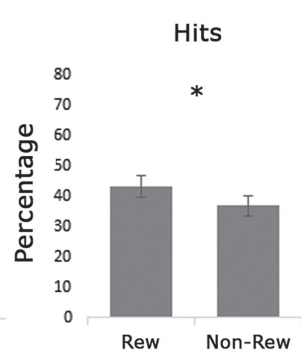
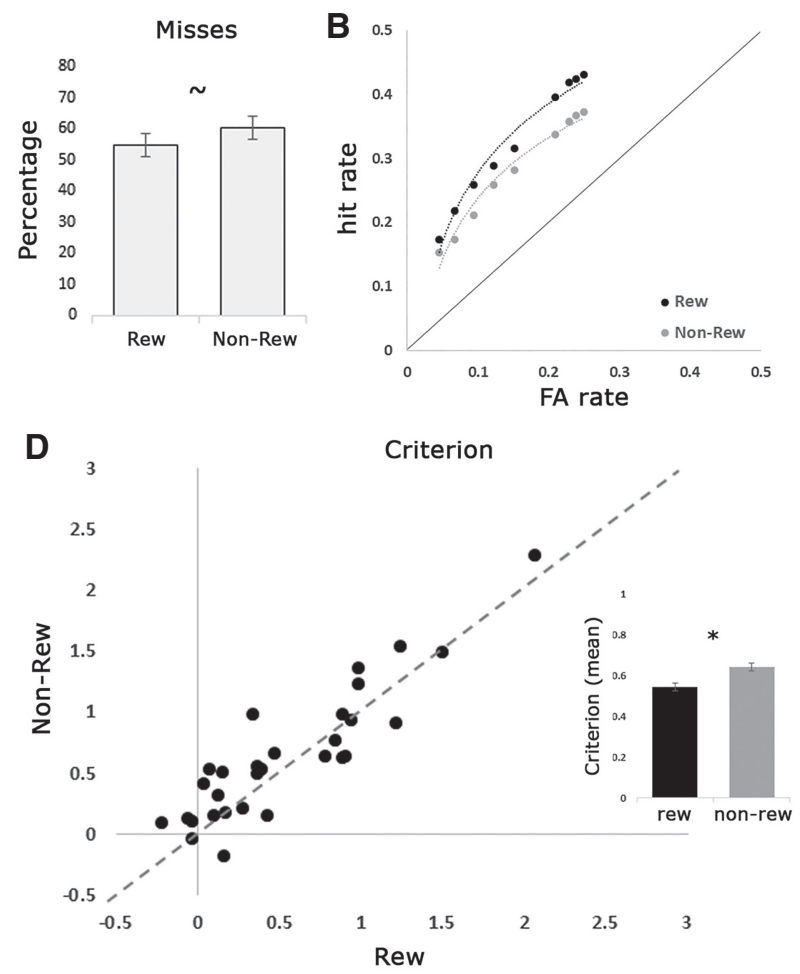

FIGURE 2. Behavioral performance. $(A)$ Memory performance for pictures presented during the free driving phase, divided into hits, misses, FA, and CR. Further division into hits for rewarded and nonrewarded items showed a preference for rewarded items $(P<0.05)$, and a concomitant decrease in misses. $(B)$ Receiver operating curves (ROC) are shown separately for items presented in rewarded versus nonrewarded contexts. Subject-by-subject values of $\mathrm{d}^{\prime}$ and criterion scores are plotted in $(C)$ and $(D)$, respectively, along with mean scores for rewarded versus nonrewarded contexts. 
initial stages of the post-rewarding driving and deteriorating thereafter, we examined whether memory for images presented within each context was affected by their order of appearance during encoding. This analysis did not yield a time-related effect on memory, suggesting that although the association between reward and context may have extinguished over time, this did not directly affect the contextual-related incidental memory effect $\left(\mathrm{BF}_{10}=0.29\right)$.

Humans and animals rapidly learn to associate between contextual cues and reinforcing stimuli (Hyman et al. 2006). In the current study, we examined the effects that associating monetary outcome with contextual cues in the form of environmental scenery during driving in a simulator, had on memory formation of incidental information presented in those contexts. We found that memory was indeed stronger for items presented in previously rewarding contexts, regardless of the particular features of the rewarded environment. Contextual cues are known to trigger behavioral and physiological responses, a phenomenon that has been extensively studied under the framework of contextual conditioning (Phillips and LeDoux 1992). Specifically, contextual details influence affective learning by serving as cues that associate affective manipulations with affective responses (LeDoux 2000). Our findings reveal an extended contextual effect on behavioral responses, demonstrating that reward-based contextual conditioning in a naturalistic setting can influence long-term memory performance of incidental information.

One conceptual framework that may account for this effect involves the interaction among learning systems, specifically those that support reinforcement learning and declarative memory (Adcock et al. 2006). Dopamine is a key candidate in this collaboration, considering its involvement in prolonging late LTP, thus leading to memory consolidation (Wittmann et al. 2005), enhancing encoding memory of novel events (Kumaran and Maguire 2006; Mather and Schoeke 2011) and allowing for the generalization of declarative knowledge (Shohamy and Adcock 2010). Additional testimonial to dopaminergic involvement in contextual learning is supported by animal studies on dopaminergic responses to rewarding contexts. In primates, midbrain dopamine neurons were shown to change their activity depending on the context, whether rewarding or not (Nakahara et al. 2004). These findings suggest that combining context with reward leads to better reward prediction. Dopamine neurons increase their firing even to unrewarding stimuli placed in previously rewarding contexts, potentially enhancing the ability to detect rewarding stimuli (Kobayashi and Schultz 2014). Whether or not these mechanisms came into play in the current study cannot at this point be concluded, though they may serve to generate predictions for follow-up investigations.

The effects of the current study resonate with the phenomenon of PIT. After forming a Pavlovian cue-reward association and a separate pairing between a particular behavior and the same reward, the mere presentation of the cue is sufficient to increase behavioral outcome, supposedly by their mutual association with reward (Lovibond 1983; Dickinson and Balleine 1994). The assigning of motivational significance to particular cues is suggested to invigorate goal-directed behavior, aimed at obtaining rewarding outcomes. Our findings indicate that assigning motivational value to a given cue set (a particular context) gives rise to enhanced incorporation of new information into long-term memory, a process that may share similar properties to the abovementioned PIT effect.

An alternative interpretation, not mutually exclusive to the above suggestions, concerns value-driven attentional effects that may come into play. Encoding of declarative information and attention are strongly linked, to the point of inseparability (Craik et al. 1996; Chun and Turk-Browne 2007). Previously rewarded stimuli are believed to capture attention and accordingly bias infor- mation processing (Anderson 2015, 2016). Accordingly, stimulus features that are learned to predict reward attract attention (Sali et al. 2014), elevating cognitive processing, among them memory formation. Notably, attention capture to previously rewarded stimuli can be carried out involuntarily (Mine and Saiki 2015). It is utterly plausible that the recruitment of the reward system in response to previously rewarded stimuli in the current task benefited encoding and subsequent memory of incidental information by increasing attention resources. We contend that the fact that no explicit instructions were given regarding neither the presentation of the images nor the ensuing memory test strengthens the ecological validity of the current findings, likening them to typical incidental memory processes of everyday occurrences. Future explorations should nevertheless examine whether similar reward-related memory effects would occur if participants were instructed to allocate attention toward the to-be-remembered items.

By assigning rewarding value to environmental context, we were able to show that an immersive experience in a rewarding context leads to superior incidental memory formation. These results imply that not only direct rewards can affect memory enhancement, but also indirect cues such as those associating reward with context in naturalistic environments.

\section{Acknowledgments}

The authors would like to thank Genela Morris and Yuval Rachely for discussions and comments regarding the manuscript.

\section{References}

Adcock RA, Thangavel A, Whitfield-Gabrieli S, Knutson B, Gabrieli JDE. 2006. Reward-motivated learning: mesolimbic activation precedes memory formation. Neuron 50: 507-517.

Anderson BA. 2015. Value-driven attentional priority is context specific. Psychon Bull Rev 22: 750-756.

Anderson BA. 2016. The attention habit: how reward learning shapes attentional selection. Ann N Y Acad Sci 1369: 24-39.

Axmacher N, Cohen MX, Fell J, Haupt S, Dümpelmann M, Elger CE, Schlaepfer TE, Lenartz D, Sturm V, Ranganath C. 2010. Intracranial EEG correlates of expectancy and memory formation in the human hippocampus and nucleus accumbens. Neuron 65: 541-549.

Bach ME, Barad M, Son H, Zhuo M, Lu YF, Shih R, Mansuy I, Hawkins RD, Kandel ER. 1999. Age-related defects in spatial memory are correlated with defects in the late phase of hippocampal long-term potentiation in vitro and are attenuated by drugs that enhance the cAMP signaling pathway. Proc Natl Acad Sci 96: 5280-5285.

Bunzeck N, Dayan P, Dolan RJ, Duzel E. 2010. A common mechanism for adaptive scaling of reward and novelty. Hum Brain Mapp 31: 1380-1394.

Chun MM, Turk-Browne NB. 2007. Interactions between attention and memory. Curr Opin Neurobiol 17: 177-184.

Craik FI, Govoni R, Naveh-Benjamin M, Anderson ND. 1996. The effects of divided attention on encoding and retrieval processes in human memory. J Exp Psychol Gen 125: 159-180.

Dickinson A, Balleine B. 1994. Motivational control of goal-directed action. Anim Learn Behav 22: 1-18.

Frey U, Morris RGM. 1998. Synaptic tagging: implications for late maintenance of hippocampal long-term potentiation. Trends Neurosci 21: $181-188$.

Gershman SJ, Daw ND. 2017. Reinforcement learning and episodic memory in humans and animals: an integrative framework. Annu Rev Psychol 68: 101-128.

Hyman S, Malenka R, Nestler E. 2006. Neural mechanisms of addiction: the role of reward-related learning and memory. Ann Rev Neursoci 29: 565-598.

Knecht S, Breitenstein C, Bushuven S, Wailke S, Kamping S, Flöel A, Zwitserlood P, Ringelstein EB. 2004. Levodopa: faster and better word learning in normal humans. Ann Neurol 56: 20-26.

Kobayashi S, Schultz W. 2014. Reward contexts extend dopamine signals to unrewarded stimuli. Curr Biol 24: 56-62.

Kumaran D, Maguire EA. 2006. An unexpected sequence of events: mismatch detection in the human hippocampus. PLoS Biol 4: 2372-2382.

LeDoux JE. 2000. Emotion circuits in the brain. Annu Rev Neurosci 23: 155-184. 
Li S, Cullen WK, Anwyl R, Rowan MJ. 2003. Dopamine-dependent facilitation of LTP induction in hippocampal CA1 by exposure to spatial novelty. Nat Neurosci 6: 526-531.

Lisman JE, Grace AA. 2005. The hippocampal-VTA loop: controlling the entry of information into long-term memory. Neuron 46: 703-713.

Lovibond PF. 1983. Facilitation of instrumental behavior by a Pavlovian appetitive conditioned stimulus. Correction to Lovibond. J Exp Psychol Anim Behav Process 9: 225-247.

Martig AK, Mizumori SJY. 2011. Ventral tegmental area and substantia nigra neural correlates of spatial learning. Learn Mem 18: 260-271.

Mather M, Schoeke A. 2011. Positive outcomes enhance incidental learning for both younger and older adults. Front Neurosci 5: 129.

Mine C, Saiki J. 2015. Task-irrelevant stimulus-reward association induces value-driven attentional capture. Atten Percept Psychophys 77: 1896-1907.

Morey RD, Rouder JN. 2011. Bayes factor approaches for testing interval null hypotheses. Psychol Methods 16: 406-419.

Murayama K, Kitagami S. 2014. Consolidation power of extrinsic rewards: reward cues enhance long-term memory for irrelevant past events. J Exp Psychol Gen 143: 15-20.

Murayama K, Kuhbandner C. 2011. Money enhances memory consolidation-but only for boring material. Cognition 119: 120-124.

Nakahara H, Itoh H, Kawagoe R, Takikawa Y, Hikosaka O. 2004. Dopamine neurons can represent context-dependent prediction error. Neuron 41: 269-280.

Pennartz CMA, Ito R, Verschure PFMJ, Battaglia FP, Robbins TW. 2011. The hippocampal-striatal axis in learning, prediction and goal-directed behavior. Trends Neurosci 34: 548-559.

Phillips RG, LeDoux JE. 1992. Differential contribution of amygdala and hippocampus to cued and contextual fear conditioning. Behav Neurosci 106: $274-285$.

Pine A, Sadeh N, Ben-Yakov A, Dudai Y, Mendelsohn A. 2018. Knowledge acquisition is governed by striatal prediction errors. Nat Commun 9: 1673.
Plancher G, Barra J, Orriols E, Piolino P. 2013. The influence of action on episodic memory: a virtual reality study. Q J Exp Psychol 66: 895-909.

Poldrack RA, Clark J, Paré-Blagoev EJ, Shohamy D, Creso Moyano J, Myers C, Gluck MA. 2001. Interactive memory systems in the human brain. Nature 414: 546-550.

Sali AW, Anderson BA, Yantis S. 2014. The role of reward prediction in the control of attention. J Exp Psychol Hum Percept Perform 40: 1654-1664.

Shohamy D, Adcock RA. 2010. Dopamine and adaptive memory. Trends Cogn Sci 14: 464-472.

Spaniol J, Schain C, Bowen HJ. 2014. Reward-enhanced memory in younger and older adults. J Gerontol B Psychol Sci Soc Sci 69: 730-740.

Takahashi H, Kato M, Takano H, Arakawa R, Okumura M, Otsuka T, Kodaka F, Hayashi M, Okubo Y, Ito H, et al. 2008. Differential contributions of prefrontal and hippocampal dopamine $\mathrm{D}(1)$ and $\mathrm{D}(2)$ receptors in human cognitive functions. J Neurosci 28: 12032-12038.

Wittmann BC, Schott BH, Guderian S, Frey JU, Heinze HJ, Düzel E. 2005. Reward-related fMRI activation of dopaminergic midbrain is associated with enhanced hippocampus-dependent long-term memory formation. Neuron 45: 459-467.

Wittmann BC, Tan GC, Lisman JE, Dolan RJ, Düzel E. 2013. Reprint of: DAT genotype modulates striatal processing and long-term memory for items associated with reward and punishment. Neuropsychologia 51: 2469-2477.

Yonelinas AP. 2001. Components of episodic memory: the contribution of recollection and familiarity. Philos Trans R Soc Lond B Biol Sci 356: 1363-1374.

Yonelinas AP, Dobbins I, Szymanski MD, Dhaliwal HS, King L. 1996. Signal-detection, threshold, and dual-process models of recognition memory: ROCs and conscious recollection. Conscious Cogn 5: 418-441.

Received May 15, 2018; accepted in revised form August 24, 2018. 


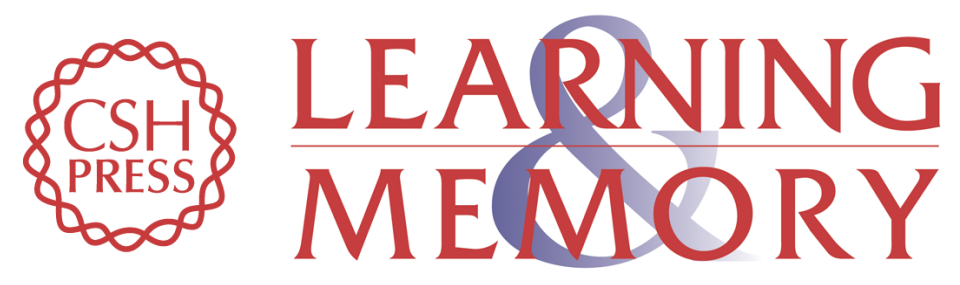

\section{Previously rewarding environments enhance incidental memory formation}

Anatoly Shneyer and Avi Mendelsohn

Learn. Mem. 2018, 25:

Access the most recent version at doi:10.1101/Im.047886.118

References This article cites 39 articles, 3 of which can be accessed free at:

http://learnmem.cshlp.org/content/25/11/569.full.html\#ref-list-1

Creative This article is distributed exclusively by Cold Spring Harbor Laboratory Press for the

Commons first 12 months after the full-issue publication date (see

License http://learnmem.cshlp.org/site/misc/terms.xhtml). After 12 months, it is available under a Creative Commons License (Attribution-NonCommercial 4.0 International), as described at http://creativecommons.org/licenses/by-nc/4.0/.

Email Alerting Receive free email alerts when new articles cite this article - sign up in the box at the Service top right corner of the article or click here. 